Mappemonde

Revue trimestrielle sur l'image géographique et les formes du territoire

$120 \mid 2017$

Varia

\title{
Un Atlas de la Nouvelle-Calédonie et de ses communes à l'heure du numérique
}

\author{
Luc Vacher
}

\section{OpenEdition}

Journals

Édition électronique

URL : http://journals.openedition.org/mappemonde/3092

DOI : $10.4000 /$ mappemonde.3092

ISSN : 1769-7298

Éditeur

UMR ESPACE

\section{Référence électronique}

Luc Vacher, "Un Atlas de la Nouvelle-Calédonie et de ses communes à I'heure du numérique », Mappemonde [En ligne], 120 | 2017, mis en ligne le 01 avril 2017, consulté le 24 septembre 2020. URL : http://journals.openedition.org/mappemonde/3092 ; DOI : https://doi.org/10.4000/mappemonde. 3092

Ce document a été généré automatiquement le 24 septembre 2020.

\section{cc) (†)}

La revue Mappemonde est mise à disposition selon les termes de la Licence Creative Commons Attribution - Pas d'Utilisation Commerciale - Partage dans les Mêmes Conditions 4.0 International. 


\section{Un Atlas de la Nouvelle-Calédonie et de ses communes à l'heure du numérique}

Luc Vacher

\section{RÉFÉRENCE}

Atlas de la Nouvelle Calédonie et dépendances (1981). Paris : ORSTOM, 226 p. ISBN 2-7099-0601-5

Louis Arréghini, Philippe Waniez. (1993). La Nouvelle Calédonie au tournant des années 1990, un état des lieux. Paris : RECLUS ; La Documentation Française ; ORSTOM, 1993, « Dynamiques du Territoire », 236 p. ISBN 2-11-002987-0 
1 Le volume Nouvelle-Calédonie de la Collection Atlas DVD de l'IRD édité en partenariat avec le Congrès de la Nouvelle-Calédonie propose, en deux DVD interactifs, une formule originale et très complète de valorisation des travaux entrepris autour de la réalisation l'Atlas de la Nouvelle-Calédonie paru en 2012.

2 «L'Atlas numérique» constitue le premier DVD et permet d'accéder à une version pdf de ce remarquable Atlas qui a mobilisé plus de 70 auteurs autour d'un vaste projet d'actualisation des savoirs géographiques sur un territoire qui a connu depuis la fin $\mathrm{du} \mathrm{XX}^{\mathrm{e}}$ siècle de profondes mutations économiques, politiques et institutionnels. Le volume, qui est plus établi dans l'esprit

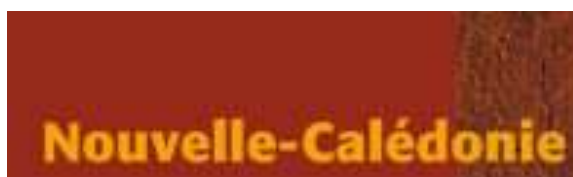

te DVo des cominunes t'atlas numérique encyclopédique de l'Atlas édité en 1981 par l'ORSTOM, que dans celui de la formule plus concise proposée par Arréghini et Waniez en 1993, dépasse très clairement le simple recueil de cartes. Il s'agit d'une synthèse très complète sur les connaissances actuelles concernant l'archipel calédonien s'organisant autour d'une soixantaine de planches cartographiques chacune accompagnée par une notice développée sur plusieurs pages et abondamment illustrée de photographies et de graphiques. Dans un format légèrement plus petit $(420$ x $300 \mathrm{~mm})$ que l'édition de 1981, la présentation s'organise, après un chapitre introductif sur le contexte régional, en 4 thèmes permettant d'aborder toutes les dimensions de la réflexion géographique sur la collectivité, ses évolutions récentes et les enjeux auxquels elle est confrontée. L'importante première partie sur les milieux s'intéresse ainsi de manière détaillée aux questions relatives à la biodiversité, aux évolutions climatiques et à celles portant sur la gestion et la préservation des milieux sensibles. La seconde partie traite des populations et de leur histoire permettant d'aborder une situation toujours en évolution sur des questions d'organisation politique et administrative, de contrôle de l'espace, de politique foncière et de relation entre les communautés. Les nouvelles dynamiques et polarités économiques autour de l'activité minière, de l'aquaculture ou du tourisme sont développées dans un troisième temps. Les auteurs nous invitent ensuite à nous pencher sur le vivre en Nouvelle-Calédonie avec des problématiques sociales (éducation, culture, sport) mais aussi à travers une approche régionale permettant une analyse de la brousse et des îles à l'échelle communale (faisant écho aux données présentées dans le deuxième DVD) mais aussi en exposant l'importante croissance urbaine du Grand Nouméa et de la zone Voh-Koné-Pouembout. 
Figure 1. Carte donnant accès aux fiches communales et permettant l'affichage d'informations générales sur la collectivité

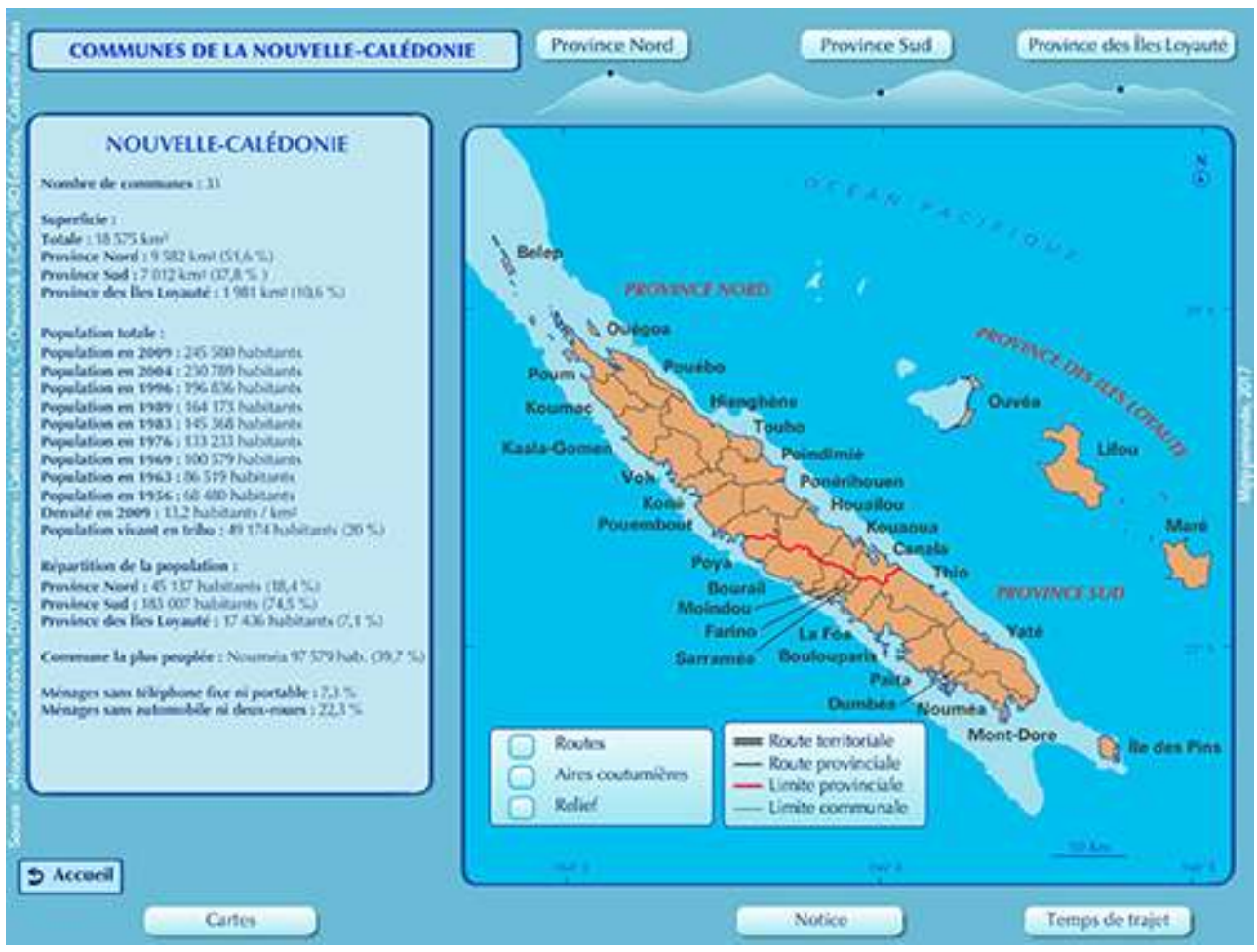

Le deuxième DVD, «Le DVD des communes ", réalisé par Céline Chauvin et JeanChristophe Gay est la pièce la plus originale de ce digipack. Il s'agit d'un DVD interactif qui donne accès à une base de données d'une très grande richesse sur les communes calédoniennes. 300 cartes originales (pour ne compter que les cartes préenregistrées) et 1500 photographies sont ainsi proposées. L'information géographique provient de la Direction des infrastructures, de la topographie et des transports terrestres (DITT) du Gouvernement de la Nouvelle-Calédonie, mais aussi d'un très important travail de terrain. Ainsi 30 à 40 photos originales sont proposées par les auteurs pour présenter la configuration spatiale et les équipements de chacune des communes. La visualisation des fiches communales se fait en sélectionnant le territoire choisi sur une carte générale ou par un menu déroulant présentant la liste des communes rassemblées par provinces (figure 1). Chaque commune est ensuite présentée dans une fenêtre ( figure 2) proposant une sélection de photos et de données statistiques produites par l'Institut de la statistique et des études économiques de la Nouvelle-Calédonie (ISEE) et une carte présentant les réseaux routier et hydrographique ainsi que l'ensemble des implantations humaines de la commune (villages, tribus, lieux-dits). À ces informations peuvent être ajoutés l'affichage des terres coutumières et privées ou le relief, il est aussi possible de compléter la carte en visualisant la localisation des sites historiques ou naturels ainsi que les écoles et centres de soins. Pour accompagner cette présentation, des ressources complémentaires sont accessibles par quatre boutons positionnés en bas de la fenêtre (figure 2). Une série de cartes communales préenregistrées ( 7 à 9 cartes suivant les communes, sur l'agriculture, l'activité minière, le statut des terres, la toponymie française, la toponymie kanak, le relief et les services) est accessible par le premier item. Chacune de ces cartes très précises est disponible dans une version écran et dans une version pdf imprimable. Un second bouton permet 
de découvrir des documents sur l'histoire et le patrimoine, généralement produits par les services « culture » des provinces. Sont ainsi présentés 13 vidéos courtes (3 à 10 minutes) et 33 documents organisés sous la forme de 1 à 3 planches d'exposition permettant d'aborder de manière richement illustrée les grands moments de l'histoire ou des témoignages sur la vie des communautés calédoniennes. Un troisième lien conduit à une notice de présentation générale de la commune rédigée par les auteurs et agrémentée d'une bibliographie. Le dernier bouton ouvre sur une carte isochrone du temps nécessaire pour accéder par la route aux différentes parties du territoire municipal depuis le chef lieu. On peut ainsi appréhender concrètement la notion de distance en Nouvelle-Calédonie en constatant par exemple que, dans la commune de Hienghene, les enfants des tribus de la Chaine comme ceux de Ouayaguette doivent compter 2 heures de route pour rejoindre le collège se situant dans le bourg à moins de 30 kilomètres à vol d'oiseau.

Figure 2. Fenêtre de présentation des communes

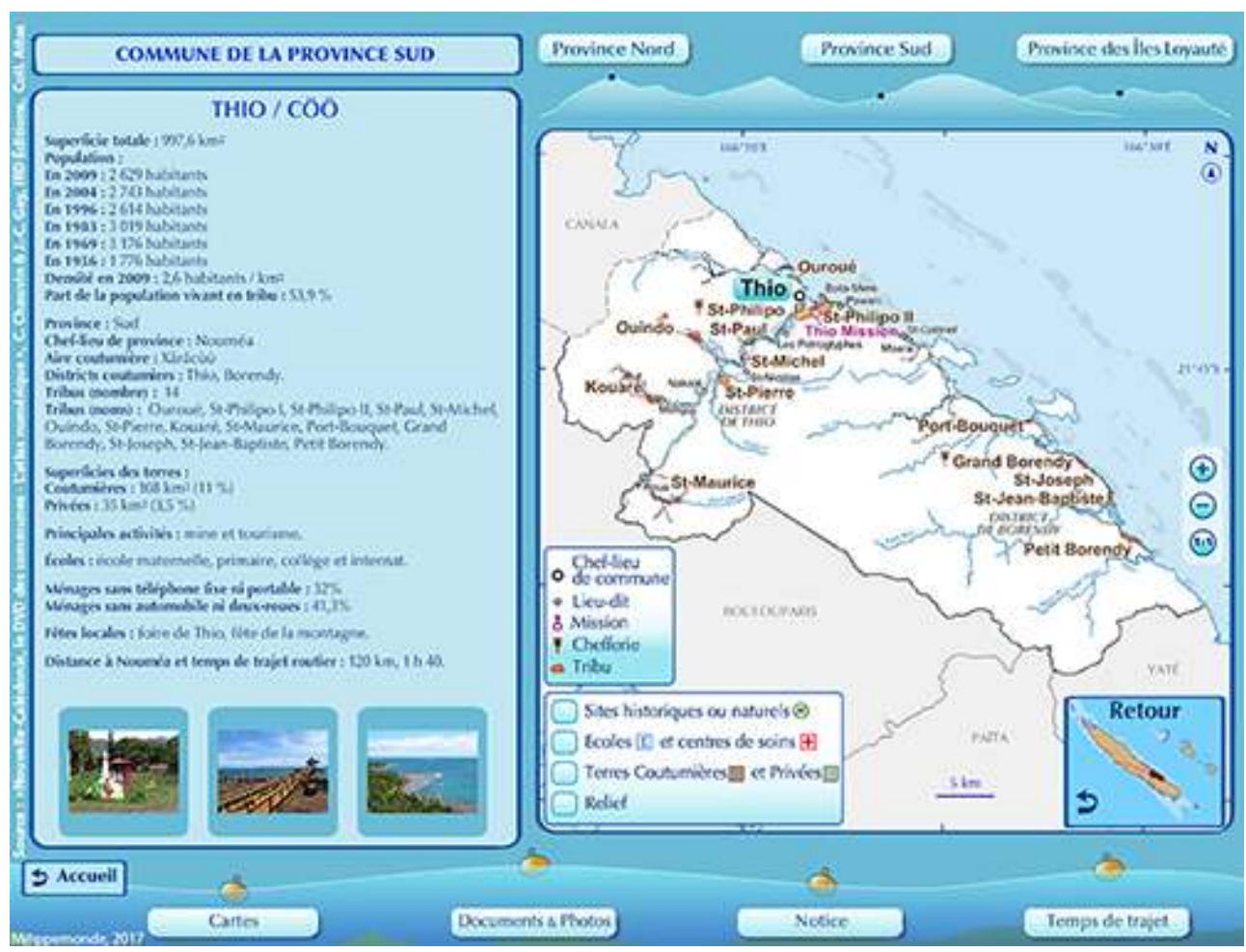

4 À la cartographie générale des territoires communaux s'ajoute une série de cartes concernant les chefs-lieux. Les informations sur ces localités se présentent sous la forme d'une nouvelle fiche interactive accessible (en cliquant sur le nom du bourg principal) depuis de la carte communale.

5 La fenêtre « chef-lieu » une fois ouverte affiche un plan présentant le réseau routier et le réseau hydrographique sur lequel sont individualisés tous les bâtiments de la localité. Ces constructions apparaissent en gris ou avec un code couleur permettant de distinguer les constructions remarquables regroupées en cinq catégories (services publics, équipements scolaires, commerces-entreprises, équipement de loisirs et santé). Sur la partie gauche de la fenêtre une liste d'une vingtaine de bâtiments sont plus précisément identifiés, ce qui permet de distinguer, à l'intérieur de ces grandes catégories, la mairie et la gendarmerie, comme de différencier le collège de l'école 
primaire. En sélectionnant l'un de ces équipements dans la liste ou en cliquant directement sur le plan, le nom et la photo de l'édifice apparaissent, permettant une immersion dans le plan digne d'un Google Street View (pas du tout d'actualité dans la brousse calédonienne) qui aurait décidé de réfléchir aux objets présentés. L'expérience pourra être complétée par l'affichage de l'orthophotographie en fond de plan. La visite s'achèvera en activant le bouton conduisant à une version imprimable du plan ou en consultant les chiffres clés de la commune grâce au lien proposé vers le site de l'Institut de la statistique et des études économiques de la Nouvelle-Calédonie (ISEE). Notons que, pour la commune de Nouméa, seul le plan du centre-ville est présenté.

Figure 3. Fenêtre de présentation des chefs-lieux de communes

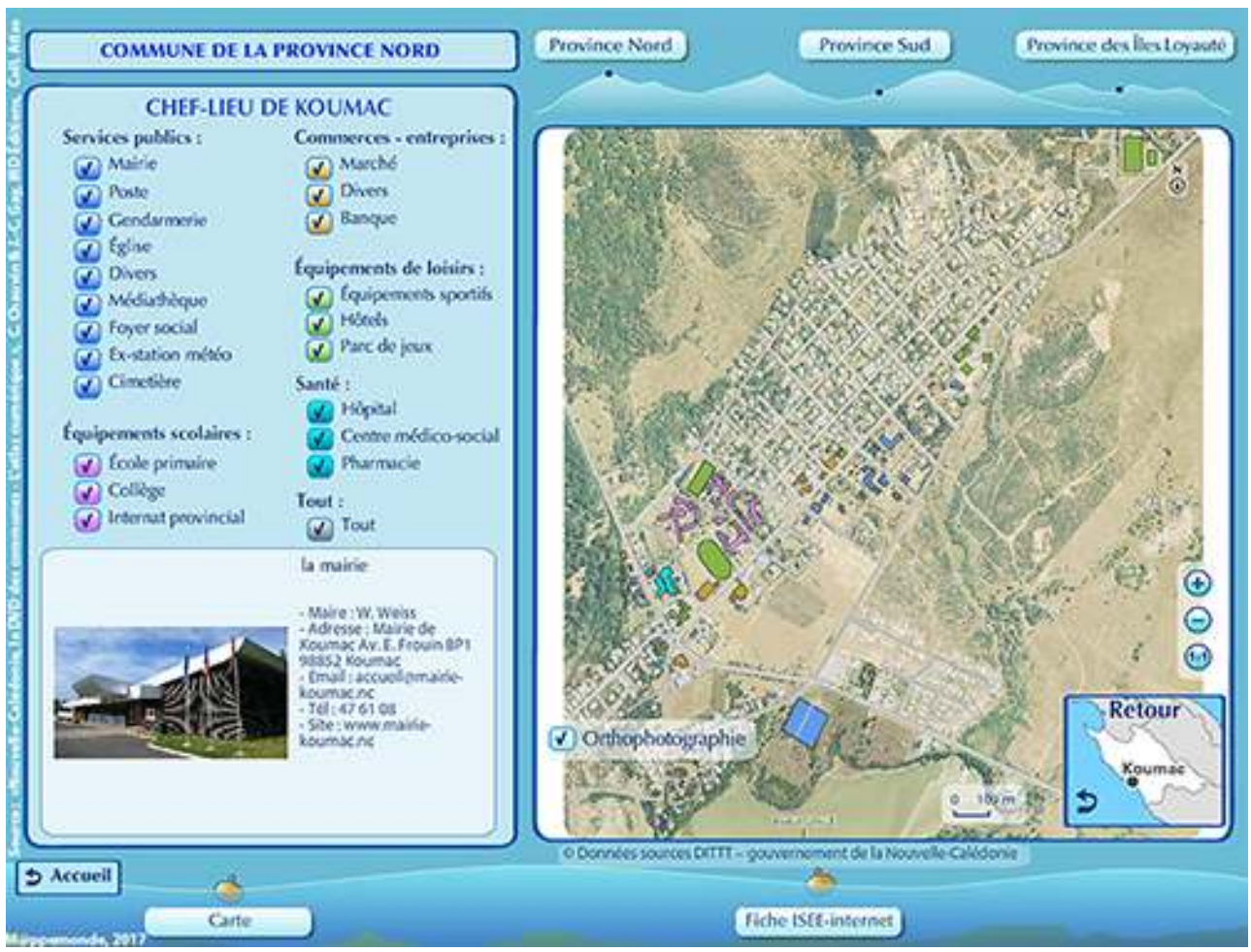

Outre la formidable somme d'informations rassemblée dans cette base de données, qui en fait un outil de référence pour tout ceux qui s'intéressent à l'étude ou à la gestion du territoire, ce CD des communes propose une approche originale de la brousse et des îles en permettant une découverte en profondeur des espaces isolés et peu accessibles. Cette visite originale permettra à bien des Calédoniens d'approfondir la connaissance de leur territoire comme elle passionnera tous ceux qui s'intéressent aux îles et à l'Océanie. En refermant ce DVD interactif, on imagine facilement un volume complémentaire qui permettrait une visualisation graphique des statistiques économiques et sociales du territoire, comme celui-ci permet de jongler avec les cartographies communales. Il est certain que les chercheurs s'empareront avec un grand plaisir de cet outil qui permet de s'interroger et d'enrichir de nombreuses réflexions sur le territoire. 


\section{BIBLIOGRAPHIE}

BONVALLOT J., GAY J.-C., HABERT É. (coord.) (2012). Atlas de la Nouvelle-Calédonie. Marseille-Nouméa : IRD ; Congrès de la Nouvelle-Calédonie, 269 p. ISBN 978-2-7099-1740-7

CHAUVIN C., GAY J.-C. (2014). Nouvelle-Calédonie, le DVD des communes. L'atlas numérique. MarseilleNouméa : IRD et Congrès de la Nouvelle-Calédonie, 2 DVD. ISBN 978-2-7099-1869-5

INDEX

Mots-clés : Nouvelle-Calédonie

\section{AUTEURS}

LUC VACHER

Géographe, Maître de Conférences HDR à l'Université de La Rochelle Equipe AGILE-UMR7266 LIENSs LIttoral ENvironnement et Sociétés CNRS-Université de La Rochelle 